\title{
Expression of prolactin receptor and response to prolactin stimulation of human NK cell lines
}

\author{
Rui SUN ${ }^{1,2^{*}}$, Ai Ling LI $^{2 *}$, Hai Ming WEI ${ }^{1}$, Zhi Gang TIAN ${ }^{1,2, * *}$ \\ ${ }^{1}$ School of Life Sciences, University of Science and Technology of China, Hefei 230027, Anhui, China. \\ ${ }^{2}$ Shandong Cancer Biotherapy Center, Shandong Academy of Medical Science, Jinan 250062, Shandong, \\ China.
}

\begin{abstract}
We have previously shown a critical role of prolactin (PRL) during maturation and anti-tumor effects of murine natural killer (NK) cells in vitro and in vivo. We extended that study by exploring the ability of human NK cell lines (NK92 and YT cell) to express PRL receptor (PRL-R) and to respond to PRL stimulation in vitro. Both human NK cell lines constitutively expressed PRL-R on membrane and mRNA transcripts, NK-92 cells contained higher level of PRL-R than YT cells, which correlated to the enhanced capacity of the cells to proliferate and to lyse target cells in response to PRL stimulation in the presence of trace amount of IL-2 or IL-15 in vitro. Two differences between IL-2 and IL-15 in functioning on human NK cells were for the first time observed. PRL synergized with IL-15 to improve proliferation of NK cells in a dose-dependent manner without double peak manifesting like IL-2. Although PRL enhanced the cytotoxicity of IL-2 or IL-15 activated NK cells, it exerted the function through up-regulating gene expression of perforin without influence of FasL in IL-2-stimulated NK cells, while in IL-15-stimulated NK cells, PRL did the function through up-regulating gene expression of both perforin and FasL but not IFN $\gamma$. PRL increased expressions of IL-2R $\alpha$ on membrane and of IL-2 mRNA in cells, indicating that PRL up-regulated NK cell function by improving positive feedback between IL-2 and IL-2R. The similar results were also observed in network between IL-15 and IL-15R. These data indicate a potential role of PRL in human NK cell modulation.
\end{abstract}

Keywords: prolactin receptor, NK cell, interleukin-2, interleukin-15.

\section{INTRODUCTION}

Prolactin (PRL), a $24 \mathrm{Kd}$ single chain hormone, is secreted by the anterior pituitary. There has been a considerable amount of literatures examining the role of PRL on immune function as well as its effects on lactation and reproduction[1-4]. Receptors with high affinity for PRL were expressed on human $\mathrm{B}$ and $\mathrm{T}$ cells by direct binding of PRL, and PRL- or IL-2-induced activation of these cells resulted in a further induction of PRL receptor expression [5-7]. PRL and the corresponding mRNA were present in human T- and B-lymphocyte as well as in B-lymphoblastoid line and $\mathrm{T}$ leukemia cells, which has been suggested to act as an autocrine growth factor for lymphoid cell prolifera-

\footnotetext{
${ }^{*}$ These authors equally contributed to this paper.

${ }^{* *}$ Corresponding author: Zhi Gang TIAN, M.D., Ph.D., Tel: 0086-551-360-7379, Fax: 0086-551-360-6783, E-mail: ustctzg@yahoo.com.cn.
}

Abbreviations: NK cells, natural killer cells; IL-2, interleukin-2; IL15, interleukin-15; PRL, prolactin; PRL-R, prolactin receptor; INF $\gamma$, interferon- $\gamma$; Fas L, Fas ligand. tion[8-11]. Specific inhibition of PRL release by bromocriptine or in the presence of anti-PRL antibodies were associated with decreased $\mathrm{T}$ cell and NK cell function[12, 13]. Interestingly, PRL increased the cellularity and antigen-specific proliferation of lymph node $\mathrm{T}$ cells of both normal and dwarf mice P[14], suggesting that the dominant effects of PRL administrated in vivo is amplification of the peripheral T-cell response. Recently, it was reported that PRL exhibited a dose-dependent enhancement upon both IgM and IgG secretion treated with anti-IgM and IL2 or anti-IgM alone in vitro $[15,16]$, indicating that PRL may affect peripheral B-cell function. PRL also increased the proliferating response and cytotoxicity of human, murine or rat NK cells to sensitive or insensitive tumor independently or synergistically with IL-2 in vitro [17-20]. We previously reported that PRL improved the hematopoiesis and lymphocyte development in syngeneic bone marrow transplantation[21-24], and exerted anti-tumor effects on tumor-bearing mice in vivo[25]. However, thus far there has been little evidence indicating that PRL can directly affect NK cell function through prolactin receptor 
on human NK cells. Here, we for the first time reported that human NK cells, using the human NK cells cells lines, might express prolactin receptors on membrane and their transcripts in human NK cells, and responded to prolactin stimulation in vitro. The molecular mechanisms underlying the prolactin stimulation, especially the relationship to IL-2 or IL-15 synergistic stimulation, were also investigated.

\section{MATERIALS AND METHODS}

\section{Cell culture and proliferation assay}

Endotoxin-free recombinant human IL-2 $\left(2.1 \times 10^{7} \mathrm{U} / \mathrm{mg}\right)$ and human IL-15 $\left(3.4 \times 10^{7} \mathrm{U} / \mathrm{mg}\right)$ were purchased from Genzyme (Cambridge, MA). Recombinant human prolactin was endotoxinfree and supplied as a gift by Dr. William J. Murphy, NCI-FCRDC, Frederick, MD, which has been used in their studies[22-25]. The IL2-dependent NK cell line NK-92, established from a patient with rapidly progressive non-Hodgkin's lymophoma[26], was purchased from ATCC and maintained in a-MEM (Hyclone) medium containing $100 \mathrm{U} / \mathrm{ml}$ IL-2 and other necessary components as described [26]. The IL-2-independnet NK cell line YT cell line, established from a patient with thymic lymphoma[27], supplied by Dr. Jiming Wang, NCI-FCRDC, Frederick, MD, was maintained in complete RPMI culture medium without adding IL-2. For proliferation assay, $\mathrm{NK}$ cells were cultured in triplicates at $37^{\circ} \mathrm{C}$ in a $5 \% \mathrm{CO}_{2}$ incubator in complete RPMI medium in 96 -well plates $\left(1 \times 10^{5}\right.$ cells $/ 200 \mathrm{ml} /$ well) in the presence of IL-2, IL-15, PRL or combinations at various concentrations. The NK cells were examined at $72 \mathrm{~h}$ after culture with cytokines by MTT color-metric method. Briefly, $5 \mathrm{mg} / \mathrm{ml}$ MTT (3-2, 5-diphenyltetrazolium bromide; Sigma) was added to all wells and the plates were incubated at $37^{\circ} \mathrm{C}$ for $4 \mathrm{~h}$, deprived of $100 \mathrm{ml}$ supernatant and $100 \mathrm{ml}$ of $10 \%$ SDS (sodium dodecyl sulphate, Sigma) were added to dissolve formazan. Results were analyzed on a BioRad Microelisa reader.

\section{${ }^{51}$ Chromium release cytotoxicity assay}

K562, a human chronic myelogenous leukemia cell line was used as target cells in cytotoxicity assay, and cultured in $15 \%$ FCS of RPMI 1640 medium. The assay was performed using NK cell lines that had been co-cultured earlier in the presence or absence of cytokines (e.g. IL-2, IL-15, PRL or combinations). The NK cells were admixed with ${ }^{51} \mathrm{Cr}$-labeled K562 target cells for NK cytotoxicity at an effector $(\mathrm{E})$ to target $(\mathrm{T})$ ratio of 10:1, 5:1, 2.5:1 and 1.25:1 as previously described[25]. After standard $4 \mathrm{~h}$ incubation, the supernatant were harvested and analyzed on a gamma counter (model 5500; Beckman Instrument, Irvine, CA). The \% specific lysis was calculated as followed: \% specific lysis $=$ CPMexp - CPMspontaneous /(CPMmaximun - CPMspontaneous) x 100\%.

\section{Reverse transcript-PCR (RT-PCR)}

The RT-PCR analysis was performed as previously described [28]. Briefly, RNA was extracted from NK cells by the acidguanidinium phenol-chloroform method. PCR primers for PRL-receptor (PRL-R), interferon-g (IFN $\gamma$ ), Fas ligand (FasL), perforin, IL2, IL-15 and $\beta$-actin were designed by us to be $18-24$ nucleotides long and to have a $100 \%$ homology with the particular regions of the genes coding characteristic extracellular regions of the molecules. The gene sequences were obtained using the OLIGO Primer Analysis Software, Version 5.0 (NBA, Software and Research Services for Tomorrow's Discoveries, National Biosciences, Plymouth, MN). PCR oligomers were produced at the Oligonucleotide Synthesis Facility of University of Science \& Technology of China. The first cDNA strand was synthesized at $37^{\circ} \mathrm{C}$ for $1 \mathrm{~h}$ with specific primer in a final volume of $20 \mathrm{~mL}$ containing $20 \mathrm{U}$ of Moloney murine leukemia virus (MMLV) reverse transcriptase, $5 \times$ reverse transcriptase buffer, 24U of RNAsin, and $0.5 \mathrm{mmol} / \mathrm{L}$ of dNTP mix. Ten microliters of first-strand cDNA was added to $20 \mathrm{~mL}$ of a PCR mix containing $1 \mathrm{~mL}$ each of upstream and downstream primers and $1 \mathrm{U}$ Taq DNA polymerase. RT-PCR was performed using a thermal cycler (Perkin-Elmer, Emeryville). PCR condition was: $95^{\circ} \mathrm{C}(1 \mathrm{~min}), 60^{\circ} \mathrm{C}$ (1 min), $72^{\circ} \mathrm{C}(1 \mathrm{~min})$, extension at $72{ }^{\circ} \mathrm{C}$ for $10 \mathrm{~min}, 30$ cycles. For amplification of PRL-R, nested RT-PCR including additional 25 cycles were performed. PCR samples were run for analysis on an ethidium bromide-stained $1.5 \%$ agarose gel and visualized by ethidium bromide staining. The band intensity of ethidium bromide fluorescence was measured using NIH Image Analysis Software Ver 1.61 (National Institutes of Health, Bethesda, MD). The intensities of the bands were determined with the use of the ratios to $\beta$-actin.

\section{Flow cytometry and immunofluorescence microscopy analysis}

For membrane staining, NK cells were suspended in ice-cold PBS containing $0.1 \%$ sodium azide and $1 \%$ FBS. The cells $\left(0.2 \times 10^{6} / 0.1\right.$ $\mathrm{ml}$ ) were then incubated on ice for $30 \mathrm{~min}$ with FITC-labeled antiCD25 (IL-2R $\alpha$ ) mAbs (10 $\mu \mathrm{g} / \mathrm{ml}$, Becton Dickinson, Mountain View, Calif.). Negative controls were cells incubated without Abs, or incubated with isotype-matched nonreactive Igs. Finally, the cells were washed twice in PBS, fixed with $1 \%(\mathrm{w} / \mathrm{v})$ paraformaldehyde/PBS solution, and analyzed by flow cytometry using FACScalibur, as previously described[21]. For immunofluorescent microscope analysis, the IL-15 or PRL were labeled by FITC using standard method, and the FITC-labeled PRL or FITC-labeled IL-15 were then used as fluorescent probe to detect PRL-R or IL-15R $\alpha$ on NK cells under fluorescent microscope.

\section{RESULTS}

\section{Constitutive expression of prolactin receptors on the membrane of human NK cell lines}

To determine the expression of prolactin and its receptor, NK-92 and YT cells, which were harvested at the logarithmic phase of growth, were examined under microscopy by direct immunofluorescence technique and using semi-quantitative RT-PCR. Analysis of immunofluorescence revealed that PRL-R constitutively expressed on the membrane of the two human NK cell lines, NK-92 (relative density $=1.71)$ expressed much higher level of PRL-R than YT cells (relative density $=0.42)($ Fig 1A), the finding was further confirmed by detecting mRNA level of PRL-R transcripts (Fig 1B).

\section{Response of human NK cells to PRL stimulation in vitro}

After confirmation of PRL-R presence on NK cells, we 
A

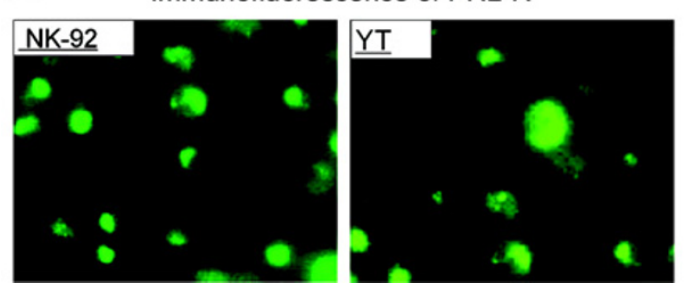

B

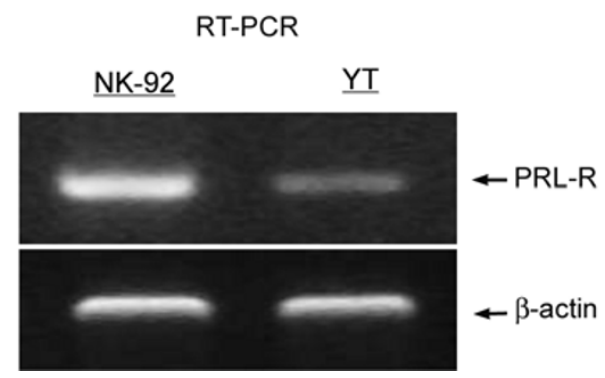

Fig 1. Prolactin receptor (PRL-R) expression on human NK cell lines. The human NK cell lines, NK-92 and YT cells, were labeled with FITC-labeled recombinant human PRL protein (FITC-PRL) and then observed under fluorescent microscopy. NK-92 cells expressed higher PRL-R positive cells than YT cells by binding to FITC-PRL (A). RT-PCR analysis was performed to further confirm the expression of PRL-R gene in human NK cell lines (B). The intensities of the bands were determined with the use of the ratios to $\beta$-actin. NK-92 cells expressed more transcripts of PRL-R mRNA than YT cells. All experiments were repeated at least four times and each sample was tripled in each experiment.

then explored the effects of PRL stimulation on NK cells. As shown in Fig 2, the PRL alone did not exert any stimulatory effects on proliferation of both human NK cell lines (Fig 2A). Interestingly, in the presence of trace amount of IL-2 or IL-15 $(5 \mathrm{U} / \mathrm{ml})$, the concentration of which did not influence NK-92 cell proliferation (Fig 2B; Fig 2C), PRL exerted stimulatory effects on NK-92 cell proliferation in a manner of two peak curve in the presence of IL2 (Fig 2D) and in the manner of dose-dependent curve in the presence of IL-15 (Fig 2E). The results indicated that PRL up-regulated NK cell function in combination with NK cell growth factors including IL-2 and IL-15. ${ }^{51} \mathrm{Cr}$ release assay and RT-PCR were performed to evaluate cytotoxicity of NK cells and cytotoxicity associated molecules expressed in NK-92 cells. The results showed that combination of PRL and IL-2 or IL-15 exerted a stronger activating effect on NK-92 cells (Fig 3). PRL significantly enhanced cytotoxicity against K562 cells of NK-92 cells in the presence of trace amounts of IL-2 (Fig 3A) $(p<0.01$ at each effector/target ratio) or IL-15 (Fig 3B) ( $p<0.01$ at each effector/target ratio). The RT-PCR analysis revealed that PRL up-regulated the expression of IFN- $\gamma$ and Perforin
A $\operatorname{rhPRL}(\mathrm{ng} / \mathrm{ml})$
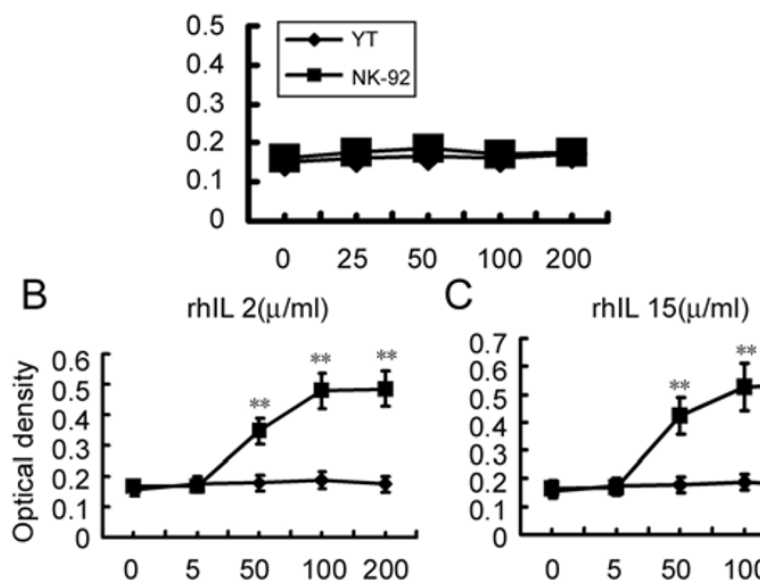

D

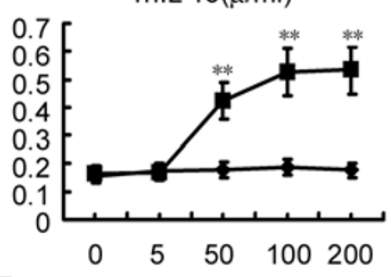

rhlL $2(5 \mu / \mathrm{ml})+$ rhPPL $(\mathrm{ng} / \mathrm{ml})$ $\mathrm{E}$
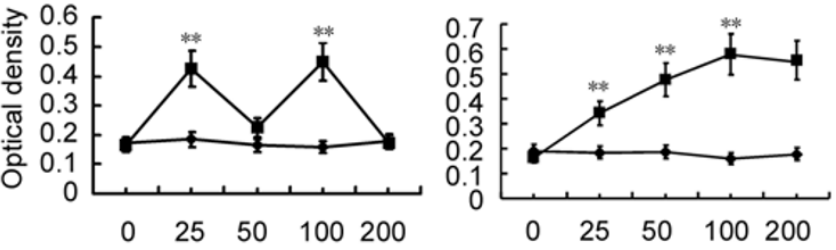

Fig 2. Proliferation of human NK cell lines in the presence of PRL and cytokines. PRL alone did not show any stimulatory effect on both cell lines (A) at concentration of $0-200 \mathrm{ng} / \mathrm{ml}$. The sub-optimal concentration of IL-2 (B) or IL-15 (C) were evaluated at $5 \mathrm{U} / \mathrm{ml}$, at which concentration NK-92 cells would not be responsive to IL-2 alone or IL-15 alone stimulation. PRL at the concentrations of 0-200 $\mathrm{ng} / \mathrm{ml}$ was used to simulate the NK-92 cells in the presence of trace amount of IL-2 or IL-15 (5 U/ml) (D, E). PRL exerted stimulating effects on NK-92 cells in a dose-dependent manner of double peak curve for PRL/IL-2 system (D) or dose-dependent curve for PRL/ IL-15 system (E). All experiments were repeated at least four times and each sample was tripled in each experiment.

but not FasL in the presence of trace amount of IL-2 (Fig 3C), in contrast, PRL up-regulated the expression of Fas$\mathrm{L}$ and Perforin but not IFN- $\gamma$ in the presence of trace amount of IL-15 (Fig 3D).

\section{PRL up-regulated NK cell functions through im- proving autocrine expression of IL-2-IL-2R or IL- 15-IL-15R network}

After it was recognized that PRL is capable of improving NK cell function in the presence of NK cell growth factor, we searched the molecular mechanisms underlying the PRL effects on NK cells. We found that NK-92 cells over-expressed IL-2R $\alpha$ (CD25) on membrane of NK92 cells after PRL stimulation using flow cytometry, but YT cell did not show any change (Fig 4A). Meanwhile, 
A rhPRL and IL2-activated NK cells

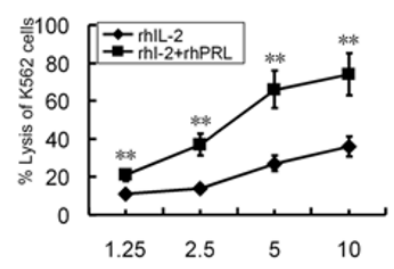

C

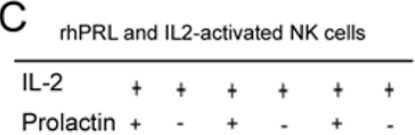

$\begin{array}{r}\text { Prolactin }+- \\ \hline \text { IFN- } \gamma \text { Fas-L Perforin }\end{array}$

$\beta$-actin
B mPRL and IL15-activated NK cells

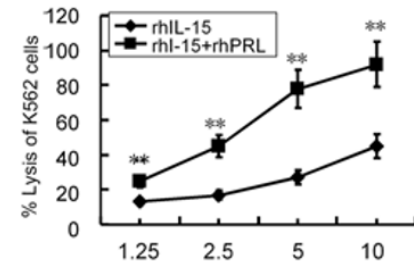

D

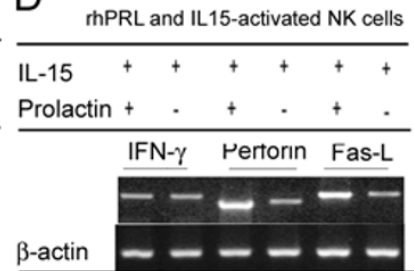

Fig 3. Cytotoxicity and cytolysis effector molecules of human NK92 cells in the presence of PRL and cytokine. NK-92 cells were harvested immediately after $12 \mathrm{~h}$ incubation in the presence of PRL $(100 \mathrm{ng} / \mathrm{ml})$ plus IL-2 $(10 \mathrm{U} / \mathrm{ml})$ or plus IL-15 $(10 \mathrm{U} / \mathrm{ml})$. The NK cells were admixed with ${ }^{51} \mathrm{Cr}$-labeled $\mathrm{K} 562$ target cells for cytotoxicity at an effector to target (E:T) ratio of 10:1, 5:1, 2.5:1 and 1.25:1 as described in Materials and Methods (A, B). The cytoplasmic RNA was extracted from NK-92 cells. The method for detecting transcripts (mRNA) of FasL, Perforin, IFN $\gamma$ and $\beta$-actin was described in Materals and Methods. The intensities of the bands were determined with the use of the ratios to $\beta$-actin $(\mathbf{C}, \mathbf{D})$. All experiments were repeated at least four times and each sample was tripled in each experiment.
A

Expression of IL-2R $\alpha$ (CD25)

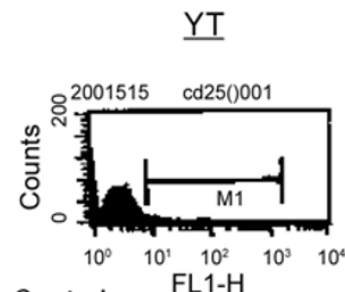

Control

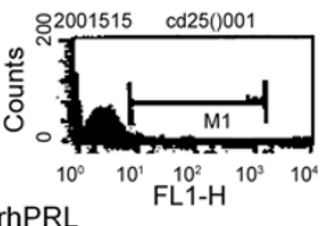

rhPRL
NK-92

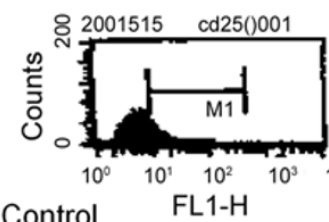

Control

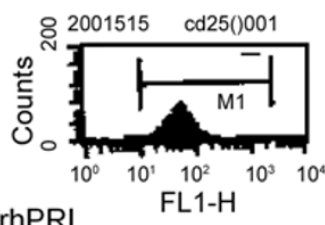

B

IL-2 mRNA expression ofNK-92

\begin{tabular}{c|cc}
\hline rhPRL & + & - \\
\hline IL-2 & & \\
\multirow{2}{*}{$\beta$-actin } & & \\
& & \\
\hline Relative density & 0.63 & 0.21 \\
\hline
\end{tabular}

Fig 4. Expression of IL-2R $\alpha$ and IL-2 mRNA in NK-92 cells after PRL stimulation. Surface expression of CD25 were increased in NK-92 cells after PRL stimulation (A). NK-92 cells were also examined for expression of IL-2 gene transcripts using RT-PCR analysis as described in Fig 1 (B). The expression of IL-2 gene transcripts was enhanced by PRL stimulation. All experiments were repeated at least four times and each sample was tripled in each experiment.

NK-92 cells increased the expression of IL-2 mRNA after PRL stimulation by RT-PCR analysis (Fig 4B), indicating that PRL stimulated expression of IL-2 receptor and their ligand (IL-2) of the same NK cells simultaneously, to form an autocrine network in the reaction. The observation on effects of PRL plus IL-2 on NK-92 cells was repeated again in PRL plus IL-15 system. The expression of IL$15 \mathrm{R} \alpha$, analyzed under fluorescent microscope after probing with FITC-labeled IL-15, was strongly enhanced on NK-92 cells after PRL stimulation (Fig 5A), and IL-15 mRNA obviously up-regulated in the cells at the same time (Fig 5B). The results may, at least partly, explain the molecular mechanisms under which the PRL up-regulated
NK cell function.

\section{DISCUSSION}

The presence of PRL-R on human NK cells is a basic indication that human NK cells are responsive to PRL stimulation. Unfortunately, until now there was only one report demonstrating that rat NK cell expressed PRL-R. Chambers et al. identified a PRL-R specific transcript using RNA isolated from highly purified, IL-2-activated rat liver NK cells by a combination of RT-PCR and Southern blotting[17]. By flow cytometric analyses, they determined that A-NK cells expressed detectable levels of PRL-R internally. Surface PRL-R was not detectable by flow- 
A

Expression of IL-15R $\alpha$ of NK-92


B

IL-15 mRNA expression of NK-92

\begin{tabular}{c|cc}
\hline rhPRL & + & - \\
\hline IL-15 & & \\
B-actin & & \\
\hline Relative density & 1.57 & 0.17 \\
\hline
\end{tabular}

Fig 5. Expression of IL-15R $\alpha$ and IL-15 mRNA in NK-92 cells after PRL stimulation. The expression of IL-2 gene was enhanced by PRL stimulation. NK-92 cells $\left(0.2 \times 10^{6} / 0.1 \mathrm{ml}\right)$ were incubated with PRL $(100 \mathrm{ng} / \mathrm{ml})$ for $24 \mathrm{~h}$, washed with PBS, and were then incubated on ice with FITC-IL-15 as described in Materials and Methods and then observed under fluorescent microscope (A). IL-15R $\alpha$ expression of NK-92 cells was significantly increased after PRL stimulation. NK-92 cells were also examined for expression of IL-15 gene using RT-PCR analysis (B). All experiments were repeated at least four times and each sample was tripled in each experiment.

cytometry on freshly isolated NK cells from normal rats, and incubation of NK cells with rIL-2 did not induce detectable surface PRL-R. Similarly, incubation of NK cells with PRL was not found to induce expression of the alpha chain of the IL-2 receptor (IL-2R $\alpha$; CD25) on NK cells [17]. We for the first time demonstrate that NK cells from human being express PRL-R, which may be important to explain the immunoregulatory function of PRL on innate immunity such as NK cell function. The characterizations of human PRL-R on NK cell lines are somewhat different from those of rat PRL-R.The PRL-R constitutively expressed on surface of human NK cell lines, but rat PRL-R was not detectable on the surface. Similarly, incubation of human NK cell lines with PRL induced up-expression of IL-2/IL-2R $\alpha$ and IL-15/IL-15R $\alpha$, but PRL did not exert any affects on expression of rat IL-2R $\alpha$. the reason for these dissimilarities is likely due to the species difference ( rat versus human) or cell status (fresh cells versus established cell lines). Interestingly, Bouchard et al. found that PRL- $\mathrm{R}^{-/-}$mice demonstrated no alterations in thymic or splenic cellularity or in the composition of the lymphocyte subsets present in primary (bone marrow and thymus) or secondary (spleen and lymph nodes) lymphoid organs. Lymphocytes from PRL- $\mathrm{R}^{-/-}$mice were functional in vitro, as they could proliferate normally to mitogens, cytokines, and allogeneic cells. PRL- $\mathrm{R}^{-/ /}$splenocytes displayed normal NK-mediated cytotoxicity to YAC-1 target cells. These results show that immune system development and function proceed normally in the absence of PRL-mediated signaling and suggest that PRL-R pathways are not essential for immunomodulation in vivo[16]. The conclusion from Bouchard et al. are obviously contrary to what have been observed in adult mice by others[1-4,12], which demonstrated that depletion of PRL-producing cells caused extensive immunosuppression of normal adult mice. It is possible that there exist some unknown mechanisms initiate to compensate for the deficiency of PRL-R during development of immune system from embryo to mature, which is possibly totally different from what has proceeded in normal adult mice.

Unlike NK-92 cells, PRL has no significant effect on the proliferation, cytotoxicity and lysis associated molecules of YT cells, which are IL-2-independent, irrespective of presence or absence of IL-2 or IL-15 (data not shown here). The double peak curve of NK cell proliferation in PRL dose response was also observed in T lymphocytes and B lymphocytes in previously studies by several laboratories[20]. The reasons for two dosage peaks of PRL action remain still unclear. Although IL-15 is well recognized to share common $\gamma$ and $\beta$ chains with IL-2, and the common chains of IL-2R system are functional mediator for finally signaling, two differences between IL-2 and IL-15 in functioning on human NK cells were for the first time observed in our study. The first difference is that PRL synergized with IL-15 to improve proliferation of NK cells in dose-dependent manner without double peak manifesting like IL-2. The second difference is that, although PRL enhanced the cytotoxicity of IL-2 or IL-15 activated NK cells, PRL exerted the function through up-regulating gene expression of perforin without influence of FasL in IL-2-stimulated NK cells. In contrast, PRL did the function through up-regulating gene expression of both perforin and FasL but not IFN $\gamma$ in IL-15-stimulated NK cells. The molecular mechanisms underlying the differences between corporation of PRL and IL-2 or PRL and IL-15 are unknown and need further investigation.

Until now, most of the researches are focusing on elucidating the mechanisms of up-regulated PRL-R expression by IL-2 stimulation to explain the function of PRL on immune cells $[4-7,11]$. We for the first time found that PRL could improve the expression of IL-2/IL-2R or IL15/IL-15R network. The results concluded that PRL, IL- 
2/IL-15, IL-2R/IL-15R and PRL-R form a network, based on which an initiator (for example, PRL, IL-2 or IL-15) may start the reaction cascade.

In conclusion, we for the first time identified PRL-R on human NK cell lines, found that human NK cells responded to PRL in double peak curve or dose-dependent manner in the presence of trace amount of IL-2 or IL-15 respectively and elucidated that the underlying mechanisms of synergistic function of PRL with IL-2 or IL-15 may be partly through up-regulating efficiency of IL-2/IL-2R or IL-15/IL-15R network. This conclusion will be helpful to explain the relationship between PRL and NK cells.

\section{ACKNOWLEDGMENTS}

This work was supported partly by Outstanding Young Scientist Award and Key Project by Natural Science Foundation of China (No.30125038, No.30230340), The Major Sate Basic research Development program of China (No.2001CB510009) and The National high technology research and Development program of China (No. 2002AA216151) by Ministry of Science and Technology of China, and Key Project by Chinese Academy of Science (No.KSCX2-2-08). The authors gratefully acknowledge the generous supply of rhPRL provided by Dr. William J Murphy of National Cancer Institute-Frederick, MD, USA, and the expert technical assistance provided by Ms. Jianhua Zhang and postgraduates Anyuan Sun and Cai Zhang in the laboratory.

Received May 30, 2003

Revised Sep 52003

Accepted Nov 28, 2003

\section{REFERENCES}

1 Matera L. Endocrine, paracrine and autocrine actions of prolactin on immune cells. Life Sci 1996; 59:599-614.

2 Murphy WJ, Rui Hallgeir, Longo DL. Effects of growth hormone and prolactin: Immune development and function. Life Sci 1995; 57:1-14.

3 Chikanza IC. Prolactin and neuroimmunomodulation: in vitro and in vivo observations. Ann N Y Acad Sci 1999; 876:119-30.

4 SM Richards, WJ Murphy. Use of human prolactin as a therapeutic protein to potentiate immunohematopoietic function. $\mathrm{J}$ Neuroimmunol 2000; 109:56-62.

5 Gala RR, Shevach EM. Identification by analytical flow cytometry of prolactin receptors on immunocompetent cell populations in the mouse. Endocrinology 1993; 133:1617-23.

6 Gagnerault MC, Touraine P, Savino W, Kelly PA, Dardenne M. Expression of prolactin receptors in murine lymphoid cells in normal and autoimmune situations. J Immunol 1993; 150:567381.

7 Prystowsky MB, Clevenger CV. Prolactin as a second messenger for interleukin 2. Immunomethods 1994; 5:49 -55.

8 Carlo R Di, Bole-Feysot C, Gualillo O, Meli R, Nagano M, PA
Kelly. Regulation of prolactin receptor mRNA expression in peripheral lymphocytes in rats in response to changes in serum concentrations of prolactin. Endocrinology 1995; 136:4713-6.

9 Matera L, Geuna M, Pastore C, Buttiglieri S, Gaidano G, Savarino A, Marengo S, Vonderhaar BK. Expression of prolactin and prolactin receptors by non-Hodgkin's lymphoma cells. Int J Cancer 2000; 85:124-30.

10 Gunes H, Mastro AM. Prolactin receptor gene expression in rat splenocytes and thymocytes during oestrous cycle, pregnancy and lactation. Cell Prolif 1997; 30:219-35.

11 Dardenne M, Moraes M do C de, Kelly PA, Gagnerault MC. Prolactin receptor expression in human hematopoietic tissues analyzed by flow cytofluorometry. Endocrinology 1994; 34: 2108-14.

12 Neidhart M. Bromocriptine has little direct effect on murine lymphocytes, the immunomodulatory effect being mediated by the suppression of prolactin secretion. Biomed Pharmacother 1997; 51:118-25.

13 Vidaller A, Guadarrama F, Llorente L, Mendez JB, Larrea F, Villa AR, Alarcon-Segovia D. Hyperprolactinemia inhibits natural killer (NK) cell function in vivo and its bromocriptine treatment not only corrects it but makes it more efficient. J Clin Immunol 1992; 12:210-5.

14 WJ Murphy, SK Durum, MR Anver, DL Longo. Immunological and haematological effects of neuroendocrine hormone: Studies on DW/J dwarf mice. J Immunol 1992; 148:3799-808.

15 P Morales, MV Carretero, H Geronimo, SG Copin, ML Gaspar, MA Marcos, J Martin-Perez. Influence of prolactin on the differentiation of mouse B-lymphoid precursors. Cell Growth Differ1999; 10:583-90.

16 B Bouchard, CJ Ormandy, JP Di Santo, PA Kelly. Immune system development and function in prolactin receptor-deficient mice. J Immunol1999; 163:576-82.

17 Chambers WH, Amoscato AA, Smith MS, Kenniston TW, Herberman RB, Appasamy PM. Prolactin receptor expression by rat NK cells. Nat Immun $1995 ; \mathbf{1 4}: 145-56$.

18 Matera L, Contarini M, Bellone G, Forno B, Biglino A. Upmodulation of interferon-gamma mediates the enhancement of spontanous cytotoxicity in prolactin-activated natural killer cells. Immunology 1999; 98:386-92.

19 Oberholtzer E, Contarini M, Veglia F, Cossarizza A, Franceschi C, Geuna M. Prolactin increases the susceptibility of primary leukemia cells to NK and LAK effectors. Adv Neuroimmunol 1996; 6:233-47.

20 Cesano A, Oberholtzer E, Contarini M, Geuna M, Bellone G, Matera L. Independent and synergistic effect of interleukin-2 and prolactin on development of T- and NK-derived LAK effectors. Immunopharmacology 1994; 28:67-75.

21 Tian ZG, Woody MA, Sun R, Welniak LA, Raziuddin A, Funakoshi S, et al. Recombinant human growth hormone promotes hematopoietic reconstitution after syngeneic bone marrow transplantation in mice. Stem Cells 1998; 16:193-9.

22 Woody MA, Welniak LA, Sun R, Tian ZG, Henry M, Richards $\mathrm{S}$, et al. Prolactin exerts hematopoietic growth-promoting effects in vivo and partially counteracts myelosuppression by azidothymidine. Exp Hematol 1999; 27:811-6.

23 Woody MA, Welniak LA, Richards S, Taub DD, Tian D, Sun R, et al. Use of neuroendocrine hormones to promote reconstitution after bone marrow transplantation. Neuroimmunomodulation 
$1999 ; 6: 69-80$

24 Welniak LA, Tian ZG, Sun R, Keller JR, Richards S, Ruscetti FW, et al. Effects of growth hormone and prolactin on hematopoiesis. Leuk Lymphoma 2000; 38:435-45.

25 Sun R, Wei HM, Zhang JH, Li AL, Zhang WC, Tian ZG. Recombinant Human Prolactin Improves Anti-tumor Effects of Murine NK cells in vitro and in vivo. Neuroimmunomodulation. 2002; 10:169-76

26 Gong JH, Maki G, KlingemannHG. Characterization of a human cell line (NK-92) with phenotypical and functional characteris- tics of activated natural killer cells. Leukemia1994; 8:652-8.

27 Nagashima S, Mailliard R, Kashii Y, Reichert TE, Herberman $\mathrm{RB}$, Robbins R, et al. Stable transduction of the interleukin-2 gene into human natural kille r cell lines and their phenotypic and functional characterization in vitro and in vivo. Blood 1998; 91: 3850-61.

28 Tian ZG, Shen X, Feng F, Gao B, IL-1 $\beta$ attenuates IFN $\beta$ induced antiviral activity and STAT 1 activation in the liver: involvement of proteasome-dependent pathway. J Immunol 2000; 165:3959-66 